\title{
Synthesis and characterization of omniphobic surfaces with thermal, mechanical and chemical stability
}

Yao Lu, Guanjie He, ${ }^{*}$ Claire J. Carmalt, and Ivan P. Parkin*

Department of Chemistry, University College London, 20 Gordon Street, London, WC1H 0AJ, UK

I.P.P.: i.p.parkin@ucl.ac.uk; G. H.: guanjie.he.14@ucl.ac.uk

A durable slippery liquid-infused porous surface (SLIPS) omniphobic coating was developed that can be simply applied to various substrates to repel water, coffee, red wine and cooking oils. The coatings retain their slippery properties and omniphobicity even after extreme thermal, mechanical and chemical tests, including rapid cooling down to liquid nitrogen temperatures $\left(-196{ }^{\circ} \mathrm{C}\right)$, heating $200{ }^{\circ} \mathrm{C}$, knife cutting, $850 \mathrm{kPa}$ Newton meter pressing, Scotch tape peeling, exposure to acid, base and corrosive salt solutions ( $\mathrm{pH}$ from 0 to 14), and neutralization on the SLIPS surface. This simple, low-cost and "ready to use" surface is believed to be useful for self-cleaning, anti-fouling, and anti-corrosion purposes potentially for large scale of industrial applications.

\section{Introduction}

Certain plants clean themselves by being water repellent such that water rolls across the surface and picks up dirt, bacteria and viruses. This has been called the Lotus effect and has inspired the production of artificial superhydrophobic (SH) surfaces ${ }^{1,2}$. To achieve the superwater-repellent ability, surfaces have to be textured (rough) and in most cases require the surface to have an intrinsic low water affinity ${ }^{3}$. However, SH surfaces usually lose their properties if contaminated by liquids whose surface tensions are lower than that of water, such as oil ${ }^{4}$. Recently SH surfaces have been specially designed from re-entrant or doubly reentrant surface roughness. These surfaces can repel water, oils and even extremely wetting liquids, such as perfluorohexane, and as such are omniphobic surfaces ${ }^{4,5}$. However, these omniphobic surfaces are not applicable for components that need lubrications such as gears and bearings ${ }^{6}$. Wong and Aizenberg et al. reported a slippery liquid-infused porous surface (SLIPS) that was inspired by the Nepenthes pitcher plant ${ }^{7,8}$. The Nepenthes-SLIPS surface is conceptually different from the Lotus-SH surface; skillfully designed roughness was used to lock lubricants at the surface so that foreign liquids/contaminants slide off from the SLIPS surfaces without staining, ${ }^{9,10}$ and thus the lubricating layer enables anti-fouling ${ }^{11-13}$, antiicing $^{14,15}$ and corrosion-prevention ${ }^{16,17}$ properties. SLIPS surfaces only function when wetted $^{18}$, which is the opposite of the 'dry' SH surfaces. However, there are still a number of limitations for the SLIPS coatings that hinder their practical applications to large scale, for example, the ease of making a SLIPS surface and its durability. ${ }^{19}$ 
The fabrication of a SLIPS surface usually involves lubricating a roughened hydrophobic surface. Lynn et al. prepared SLIPS surfaces through lubricating a hydrophobic multilayer, which was fabricated by 35 repeated cycles of chemical bath treatments. The prepared SLIPS surface guided a water droplet travelling within a designed path ${ }^{20}$. Tesler et al. fabricated robust SLIPS surfaces on steel by electrodeposition of nanoporous tungstite films followed by lubrication with Krytox GPL-K103 ${ }^{21}$. Using anodization, Wang et al. created a textured surface morphology on aluminium substrates, which became a SLIPS surface after being fluorinated and then lubricated ${ }^{22}$. Zhang et al. deposited fluorinated silicone nanofilaments on glass surfaces to make SH surfaces, and then SLIPS surfaces were fabricated by lubricating the SH surfaces. The prepared SH and SLIPS surfaces could be reversibly interconverted through addition and evaporation of the lubricating layer ${ }^{23}$. Hao et al. prepared SLIPS surfaces by lubricating a Teflon membrane with Dupont Krytox 103, 105 or $107 .{ }^{24}$ Although the aforementioned methods could successfully fabricate SLIPS surfaces and achieve certain properties, large scale production and applications would be challenging due to high cost, complex fabrication, limited size and the nature of the substrates. For example, anodization is largely limited to coat metallic materials; too many processes in the surface fabrication of non-metallic materials would increase the cost etc. Therefore, a general method of coating SLIPS surfaces on different substrates via a scalable method is required.

Durability of a surface is also an important factor in practical conditions, and this can be divided into three aspects - thermal stability, mechanical robustness and chemical durability. Thermal stability of SLIPS surfaces have been studied at high and low temperatures. To test how SLIPS surfaces could survive and retain omniphobic properties at high temperatures, two strategies are normally employed - one is to test the mobility of liquid droplets after cooling down from a high temperature ${ }^{25}$; the other is to test the omniphobicity of the SLIPS surfaces at elevated temperature ${ }^{26}$. For low-temperature studies, anti-icing tests were carried out at $-4{ }^{\circ} \mathrm{C},{ }^{7}-10{ }^{\circ} \mathrm{C},,^{27,28}-15{ }^{\circ} \mathrm{C},{ }^{29}-20{ }^{\circ} \mathrm{C}^{13}$ etc. with a cooling rate of $\sim 2{ }^{\circ} \mathrm{C}$ per second. Although these surfaces maintained slippery properties after defrosting, few reports have shown that the SLIPS surfaces were able to retain omniphobicity after defrosting from a rapid cooling down to an extremely low temperature (e.g. liquid nitrogen). Mechanical weakness of a liquid repellent surface usually hinders its practical applications because the micro and nano scaled surface morphology can be easily damaged even sometimes by brushing with a tissue. Some robustness tests on omniphobic surfaces were previously reported such as knife scratches $^{7}$ and particle abrasion ${ }^{30}$. However, the mechanical durability of a coating was difficult to quantify via these tests. Chemical durability gives an indication of the stability of a surface when it meets corrosive liquids, such as acids and bases ${ }^{31,32}$. In order to fabricate chemical durable SLIPS surfaces, neither the lubricating layer nor the substrate should react with acids and bases. Although many reported SLIPS surfaces showed that they could function at various temperatures, after mechanical damage or after coming into contact with acids and bases, few reports have demonstrated that the fabricated SLIPS surfaces could address thermal, mechanical and chemical durability under even more rigorous conditions, those that are applicable to real world applications. 
A SH paint that was made of dual-scaled $\mathrm{TiO}_{2}$ nanoparticles and perfluorooctyltriethoxysilane has been previously developed to be treated on various substrates. ${ }^{6}$ Here, we show how the SH paint can be transformed into a slippery state by adding a layer of lubricant. The prepared SLIPS coatings can be treated on different substrates to make these materials omniphobic - repelling water, coffee, red wine and cooking oil. Thermal stability was studied at extreme temperatures such as $-196{ }^{\circ} \mathrm{C}$ in liquid nitrogen and at $200{ }^{\circ} \mathrm{C}$, in addition, the relationship between heating time and slipperiness was also tested. We show mechanical robustness using knife scratches and further quantify the robustness of the SLIPS surface by measuring sliding angles (SA) after adding different vertical forces onto the surfaces and a Scotch tape peeling test. The treated slippery surfaces demonstrated chemical durability by repelling water droplets with $\mathrm{pH}$ ranging from 0 to 14 , and further we show acid-base neutralization of droplets on the SLIPS surface with $\mathrm{pH}$ of 0 and 14. In addition, corrosive salt solution was also used for chemical etching tests. Finally, we tested an individual SLIPS sample with all the extreme conditions from our thermal, mechanical and chemical durability tests, and the sample retained omniphobically slippery properties even after all these tests.

\section{Results and discussion}

Figure 1 (a) shows the two-step process used in making a SLIPS surface on a given substrate. In Step 1, the SH paint that was made of dual-sized $\mathrm{TiO}_{2}$ nanoparticles and fluorosilane ${ }^{6}$ was treated on the substrates (glass or filter paper) via dip-coating. In Step 2, one of the lubricating oils (Fluorinert FC-70, Krytox 100B or Krytox 104A) was dropped on the painted SH surface (Supplementary Table S1). The lubricant drops spontaneously wetted and spread on the SH surface and formed a lubricating layer to make the surface slippery. FC-70 was used throughout the experiments unless otherwise specified. Figure 1 (b) and (c) shows the scanning electron microscopy (SEM) images and the X-ray diffraction (XRD) patterns of the SH paint in Step 1, respectively. SEM images show that the SH paint consists of dual-scale nanoparticles that make the surface porous; Supplementary Fig. S1 shows a cross-sectional SEM image of a SH paint treated glass slide, and the thickness of the SH coating was $\sim 14 \pm 1$ $\mu \mathrm{m}$. XRD patterns of these nanoparticles were indexed to the standard anatase phase of $\mathrm{TiO}_{2}$ (JCPDS No. 21-1272). Figure 1 (d) and (e) represent the FTIR and Raman spectra of the lubricated surface and the SH surface respectively. There are slight differences in the FTIR spectroscopy for the two surfaces from $920-1420 \mathrm{~cm}^{-1}$. The peak at $\sim 1240 \mathrm{~cm}^{-1}$ corresponds to the carbon $s p^{3}$ bound to nitrogen and the peaks at $\sim 1308, \sim 1206$ and $\sim 1139 \mathrm{~cm}^{-1}$ are from C-F stretching of FC-70. ${ }^{33,34}$ Raman spectra of the two surfaces are similar with the same three main labelled peaks $\left(396.4,515.8\right.$ and $\left.637.5 \mathrm{~cm}^{-1}\right)$ in Fig. 1 (e), which are assigned to Ti-O stretching type vibrations. ${ }^{35}$

This coating can be simply treated on various substrates to make SLIPS omniphobic (repels water and oil) surfaces. In this work, we used glass slides and filter papers as substrates (all the tests were performed on glass substrates unless otherwise specified). Figure 2 (a) shows a liquid dropping test on the SLIPS glass substrate (fabricated via dip-coating with the SH paint 
followed by FC-70 lubricating) with water (dyed blue), coffee, red wine and corn oil. Supplementary Movie S1 shows that the four liquids slid off the treated slippery parts and then contaminate the untreated parts. Figure 2 (b) and Supplementary Movie S2 show that all the tested liquids slid off the SLIPS filter papers (fabricated via syringe-extruding of SH paint and then FC-70 lubricant), indicating that the treated filter papers were slippery and omniphobic. The aforementioned tests showed individual liquids dropped on tilted SLIPS surfaces; if given more contact time with different liquids the SLIPS surface remains selfcleaning as shown in Supplementary Fig. S2 and Supplementary Movie S3. Some practical conditions were examined by looking at how common liquids found in the kitchen interacted with the SLIPS surface (e.g. tomato ketchup, cooking oil), or running and continuous liquids (e.g. poured oil spills or running tap-water). Supplementary Fig. S3 shows that the fabricated SLIPS surface repelled tomato ketchup (Supplementary Movie S4); running corn oil and water from a tap (Supplementary Movie S5).

To guarantee the self-cleaning/anti-fouling properties, good durability and the ability to recover are important especially when the surfaces meet extreme conditions, such very low or high temperatures. Instead of using a fridge or a cooling chamber to decrease the surface temperatures at a cooling rate of $\sim 2{ }^{\circ} \mathrm{C}$ per second as has been used in previous work, ${ }^{7,14,27-29}$ we performed a more aggressive method using liquid nitrogen. Figure 3 (a) and Supplementary Movie S6 show a SLIPS surface after immersion into liquid nitrogen $\left(-196^{\circ} \mathrm{C}\right)$ to test its durability and recovery. Coffee, red wine and corn oil were dropped on the surface and it was observed that the droplets froze after a few seconds. Water was then added to aid melting and all the liquids slid off, after the surface had warmed, indicating that the SLIPS surface recovered. Figure 3 (b) and Supplementary Movie S7 show that water and oil droplets were positioned on a (104A lubricated) SLIPS surface which had been heated for $1 \mathrm{~min}$ at $200{ }^{\circ} \mathrm{C}$. The water-oil mixture was then fried to splash, and finally slid off and the SLIPS surface remained clean. In order to test the relationship between slippery properties and heating time, we measured contact angle hysteresis (CAH) of SLIPS samples (3 samples lubricated by FC-70, 100B and 104A respectively) before and after heating for every 5 mins at $100{ }^{\circ} \mathrm{C}$. Figure $3\left(\mathrm{c}_{\mathrm{i}}\right)$ shows CAH-heating time on the SLIPS surfaces (FC-70 and 100B), respectively. At $0 \mathrm{~min}$, the four liquids could easily slip off from both FC-70 and 100B lubricated SLIPS surfaces in a slippery state with a small CAH (below $5^{\circ}$ ). Then the lubricants evaporated after 5 minutes of heating; water and water-based liquids (coffee and red wine) could roll off easily and the CAH remained below $10^{\circ}$; while corn oil was "pinned" on the surface with a CAH greater than $30^{\circ}$, indicating that this surface could only repel water but not oil, showing a typical SH state. The thickness of the lubricating layer was characterized by measuring the weight of added lubricants on a given area. It has been shown that the transition between SLIPS and SH states can be induced by evaporation and relubrication $^{23}$, however, in order to test the surface thermal durability, multiple cycles of transitions must be tested. Figure $3\left(\mathrm{c}_{\mathrm{ii}}\right)$ shows the CA of water as the sample was lubricated (with FC-70), the lubricant was removed by evaporation and re-lubricated for multiple cycles. Supplementary Table S2 shows the water contact angle (CA), weight and lubricating layer's thickness in the multi-cycle reversible transition tests. The surface returned to $\mathrm{SH}$ state when the lubricants were evaporated, while it retained its slippery character when the dry SH 
surface was lubricated again, even after more than 10 cycles. Supplementary Movie S8 shows liquid dropping tests on the sample that was tested over 10.5 evaporation-recharge cycles, when the surface was lubricated after 10 cycles of evaporation, using water, red wine, coffee, corn oil and hexadecane, respectively, indicating that the surface remained slippery and omniphobic. Figure $3\left(\mathrm{c}_{\mathrm{iii}}\right)$ and $\left(\mathrm{c}_{\mathrm{iv}}\right)$ shows the environmental scanning electron microscope (ESEM) images of FC-70 and 100B lubricated samples after heating for 5 min at $100{ }^{\circ} \mathrm{C}$, respectively. These samples show a dry and chapped surface morphology compared with the untested SLIPS samples (Supplementary Fig. S4), indicating that the lubricants of FC-70 and 100B were removed from the SH paint due to evaporation. Figure $3\left(\mathrm{~d}_{\mathrm{i}}\right)$ shows the plots of CAH-heating time on 104A lubricated SLIPS surfaces. All the CAH were below $10^{\circ}$ from 0 to 35 min heating, indicating that the use of a lubricating layer with a higher viscosity and estimated useful range of temperature would achieve a better thermal durability for SLIPS surfaces under high temperatures. In particular, the lubricant no longer evenly covered the SH substrate after 35 min heating, instead, some parts on the heated sample showed less lubricant than the other areas, we then distinguished the two different areas using 'dry' and the 'wet' parts. Here, the 'dry' part refers to the area with less lubricant while the 'wet' part refers to the area with more lubricant. The CAHs on the 'dry' and the 'wet' parts respectively were between $2^{\circ}-8^{\circ}$ and did not show significant differences, indicating that even a thin layer on the dry 'part' would still achieve a slippery state. Supplementary Table S3 shows the mass of samples and the average thickness of lubricants with the SLIPS-heating time tests. Figure 3 $\left(\mathrm{d}_{\mathrm{ii}}\right)$ shows the ESEM image of $104 \mathrm{~A}$ lubricated samples after heating for $5 \mathrm{~min}$ at $100{ }^{\circ} \mathrm{C}$, the surface retained the lubricating layer and did not significantly change from the untested 104A lubricated sample (Supplementary Fig. S4), and this is why the surface retained slippery in contrast to the FC-70 and 100B lubricated samples.

Mechanical robustness is a key factor for self-cleaning surfaces to be used in practical conditions. We used knife cut, Newton meter press and Scotch tape peeling methods to test how these SLIPS samples would tolerate mechanical impacts and retain their omniphobicity (in the mechanical robustness tests, coatings were bonded using double-sided-tapes, see Methods). Figure 4 (a) shows the cutting process and Supplementary Movie S9 shows the knife cut of FC-70, 100B and 104A lubricated samples and the subsequent liquid dropping tests, indicating the surfaces retained repellence to corn oil, red wine, coffee, and water. Figure 4 (b)-(d) shows the ESEM images of knife scratched (b) FC-70, (c) 100B and (d) 104A lubricated samples. Scratches were found to be filled in by lubricating oils, so that the surfaces retained omniphobicity after the knife cut tests.

However, knife cut tests only impacted a small area on the sample surfaces, and it would be easier for the surfaces to recover. To further evaluate the mechanical robustness of the SLIPS surface over a larger area, we used a Newton meter to press the coated surfaces and to test the pressed area with water and corn oil as shown in Figure 4 (e)-(g) and Supplementary Movie S10. We measured water and oil SAs of surfaces after each press (forces ranging from $2.6 \mathrm{~N}$ to $175 \mathrm{~N}$ at an area of $2.06 \times 10^{-4} \mathrm{~m}^{2}$, forces larger than $175 \mathrm{~N}$ would break the glass substrates) as shown in Figure $4(\mathrm{~h})-(\mathrm{j})$. At higher pressure, the water and oil SAs got larger but were still below $2.5^{\circ}$ for the FC-70 and 100B lubricated samples and below $10^{\circ}$ for the 
104A lubricated sample. The SAs were larger on 104A lubricated samples than those on the FC-70 and 100B lubricated samples, however, even the largest SA was below $10^{\circ}$, indicating that these surfaces would tolerate the impact of these forces and would remain slippery. Supplementary Fig. S5 shows the ESEM images of FC-70, 100B and 104A lubricated SLIPS surfaces after being pressed to $\sim 850 \mathrm{kPa}$. On the FC-70 sample, the footprint of the press was seen; however the morphologies showed little difference between the inside and outside of the footprint indicating that even after the press, the coating was still fixed on the substrate by the tapes. This is why the surface still repelled both water and oil after being pressed. On the $100 \mathrm{~B}$ and $104 \mathrm{~A}$ lubricated samples after $\sim 850 \mathrm{kPa}$ mechanical press, traces of the press were not discovered as the lubricating layers covered the surface structures, however, in the case of the FC-70 sample, it can be inferred that the surface structures would not greatly change. Hence, these surfaces retain their slippery and omniphobic properties with unchanged surface morphologies and the defect-free lubricating layers even after a vertical press of $\sim 850 \mathrm{kPa}$.

Scotch tape peeling was also used to test the surface robustness. In this method, the Scotch tape was firstly adhered to the sample surface and then removed. Supplementary Table S4 shows sliding angles of both water and corn oil on the FC-70, 100B and 104A lubricated SLIPS surfaces before and after the Scotch tape peeling. After this test, the sliding angles were still small and both water and corn oil droplets were repelled by the surfaces, indicating that these samples retain omniphobic properties after the Scotch tape peeling test.

Chemical durability of the SLIPS surfaces was tested using acid and base droplets with $\mathrm{pH}$ values from 0 to 14. Figure 5 (a) shows that the FC-70, 100B and 104A lubricated samples that were firstly mechanically scratched by a knife, and then water droplets with different $\mathrm{pH}$ values were dropped onto these scratched surfaces (tilted $\sim 28^{\circ}$ ). All the FC-70, 100B and $104 \mathrm{~A}$ lubricated samples were clean after the tests, indicating that these surfaces are chemically stable. Acid and base neutralization was further carried out on the SLIPS surfaces. Figure $5(\mathrm{~b})$ shows acid $(\mathrm{pH}=0)$ and base $(\mathrm{pH}=14)$ droplets were positioned onto a FC-70 lubricated surface, the two droplets were guided to combine and neutralize [Fig. 5 (c)], and finally slid off from the SLIPS surface [Fig. 5 (d)]. Supplementary Movie S11 shows the acid-base neutralization on FC-70, 100B and 104A lubricated samples. All the samples remained clean and slippery after the tests. Supplementary Fig. S6 shows the ESEM images of the samples after the acid-base neutralization test. Compared with the untested samples (Supplementary Fig. S4), the surface morphologies did not significantly change indicating that $\mathrm{pH}$ did not physically influence the prepared SLIPS surfaces. This is due to the selected lubricants being immiscible with acids and bases, hence the $\mathrm{SH}$ substrate covered with fluorosilane, that is also stable to either acid or base ${ }^{36}$, created SLIPS surfaces that were chemically durable to acids and bases.

To further test the chemical stability of the SLIPS samples, corrosive salt solution was used to etch the sample substrates. A solution with $\mathrm{Cu}^{2+}$ usually reacts with mild steel; this etching process was even used to create surface micro-nano morphologies. ${ }^{37,38}$ Supplementary Fig. S7 shows that droplets of $0.5 \mathrm{~mol} \mathrm{~L}^{-1} \mathrm{CuSO}_{4}$ aqueous solution were positioned onto untreated mild steel and 104A lubricated SLIPS mild steel, respectively. After 10 minutes, the 
untreated sample was chemically etched, while the SLIPS coated mild steel did not show any traces of corrosions, indicating the SLIPS coating is chemically stable.

These surfaces have shown their durability and retained slipperiness after individual thermal, mechanical and chemical tests. However, in practical circumstances, a surface would suffer extreme temperatures, together with severe mechanical impacts and corrosive chemicals. Here we subjected one single sample (104A lubricated, the coating was bonded on the glass substrate using double-sided-tape) to all these tests as shown in Supplementary Fig. S8. The sample was heated at $200{ }^{\circ} \mathrm{C}$, scratched, pressed at $175 \mathrm{~N}$, and then acid and base were positioned on the sample to neutralize. After these tests, a corn oil droplet was still able to slide off, indicating the surface retained slippery and omniphobic properties. ESEM image showed that the surface did not significantly change after these tests, indicating that the SLIPS surface is thermally, mechanically and chemically durable.

\section{Conclusions}

In conclusion, we developed a durable omniphobic SLIPS coating that can be simply treated on various substrates, such as glass and paper. The surface retained repellence to water, coffee, red wine and corn oil even after being treated with extremely cold (liquid nitrogen, $\left.196^{\circ} \mathrm{C}\right)$ and hot temperatures $\left(200^{\circ} \mathrm{C}\right)$. By selecting the viscosity and estimated useful range of the lubricants, we can achieve either a thermally stable SLIPS surface or a reversible transition between SH and SLIPS states. In the mechanical robustness tests, the lubricants partially covered the scratch traces so that the surfaces retained slippery. Further, SAs of water and oil were measured on the SLIPS surfaces before and after vertical Newton meter press at forces from $2.6 \mathrm{~N}$ to $175 \mathrm{~N}(\sim 850 \mathrm{kPa})$ and a Scotch tape peeling test, the SAs did not significantly change indicating that the SLIPS surfaces were mechanically robust. In the chemical tests, the surfaces repelled water droplets with $\mathrm{pH}$ from 0 to 14 . We also presented acid-base neutralization on the SLIPS surface and the neutralized droplet could easily slide off. We further showed that the SLIPS coating was able to protect mild steel from being etched by $0.5 \mathrm{~mol} \mathrm{~L}^{-1} \mathrm{CuSO}_{4}$ aqueous solution. Finally we showed all the thermal $\left(200{ }^{\circ} \mathrm{C}\right.$ heating), mechanical (scratch and $850 \mathrm{kPa}$ press) and chemical (acid and base dropping and neutralization) tests on one single sample, and the surface retained repellence to acid, base and oil droplets. This coating could be treated on various substrates with extreme thermal, mechanical and chemical durability, and it is believed to be useful for self-cleaning, antifouling, and anti-corrosion purposes in a large scale of industrial applications.

\section{Experimental section}

\section{Materials and fabrication}

$1 \mathrm{H}, 1 \mathrm{H}, 2 \mathrm{H}, 2 \mathrm{H}$-perfluorooctyltriethoxysilane (fluorosilane), $\mathrm{TiO}_{2}$ nanoparticles and Fluorinert FC-70 were purchased from Sigma-Aldrich. $\mathrm{TiO}_{2} \mathrm{P} 25$ was purchased from Degussa. Krytox 100B and Krytox 104A were kindly donated by GBR Technology Limited, UK. SH paint was made according to our previous recipe, which is to add $1.00 \mathrm{~g}$ of $1 \mathrm{H}, 1 \mathrm{H}$, 
$2 \mathrm{H}, 2 \mathrm{H}$-perfluorooctyltriethoxysilane into $99 \mathrm{~g}$ of absolute ethanol, and mechanically stir for 2 hours. $6 \mathrm{~g}$ of $\mathrm{TiO}_{2}$ nanoparticles (Aldrich) and $6 \mathrm{~g}$ of Degussa $\mathrm{P} 25 \mathrm{TiO}_{2}$ were added to the resulting solution to make a paint. ${ }^{6}$

The paint was treated on glass slides via dip-coating $(120 \mathrm{~mm} / \mathrm{min})$ and on filter paper via syringe-extruding of the paint. One of the lubricating oils was then dropped on the SH paint via a syringe to make a liquid infused layer. The layer thickness was calculated by measuring the weight and density of these lubricating drops at room temperature on a given area.

\section{Characterizations}

The samples before lubricating were characterized by a scanning electron microscope (SEM, JEOL JSM-6700F), and the samples after lubricating were characterized by an environmental scanning electron microscope (ESEM, Philips XL30), X-ray diffractometer (XRD, D4 ENDEAVOR, $\mathrm{Cu}-\mathrm{Ka}$ radiation), attenuated total reflectance Fourier transform infrared spectroscopy (ATR-FTIR, BRUKER, platinum-ATR), and Raman spectroscopy (633 nm laser wavelength). Contact angle (CA) was measured at ambient temperature via the sessiledrop method using an optical CA meter (FTA 1000). Contact angle hysteresis (CAH) was calculated as the value of advancing $\mathrm{CA}\left(\theta_{\mathrm{a}}\right)$ minus receding $\mathrm{CA}\left(\theta_{\mathrm{r}}\right)$, which were measured via two methods, i.e. tilting-plate goniometry (TPG) and captive-drop goniometry (CDG) ${ }^{39}$ In TPG, when a droplet just rolls off as the surface is tilted (here the tilted angle of the surface is called sliding angle, SA), the downhill side CA is $\theta_{\mathrm{a}}$ while the uphill side CA is $\theta_{\mathrm{r}}$. In the case that a droplet was pinned onto a surface, the advancing and receding contact angles were measured when the surface was tilted at $90^{\circ}$. In $\mathrm{CDG}$, a droplet is positioned on the surface with a needle connected to a syringe. $\theta_{\mathrm{a}}$ is read via slightly filling the droplet and $\theta_{\mathrm{r}}$ is read by removing liquid from the droplet. Here, CAH of SLIPS and SH surfaces were measured using TPG and CDG methods, respectively, according to the feasibility of the experiments.

\section{Liquid dropping tests}

Contamination repellent tests were carried out on $\sim 22^{\circ}$ tilted SLIPS glass and filter paper substrates, respectively. Four liquids included water, coffee, red wine and corn oil. Ketchup and hexadecane were also involved in some of the tests. Water: distilled, dyed with methylene blue to aid visibility and this does not change its behaviour. Coffee: Nescafe instant; $11 \mathrm{~g}$ of coffee powder was mixed with $300 \mathrm{~mL}$ of water. Red wine: $12.5 \% \mathrm{Vol}$. Corn oil: Mazola pure corn oil, from Waitrose, London. Tomato ketchup: from McDonald.

\section{Thermal stability tests}

1. Recovery tests after extremely cold environment. A SLIPS glass surface was dipped into liquid nitrogen for $\sim 3 \mathrm{~s}$ and then frozen. Coffee, red wine, corn oil and water were respectively dropped on the surface to test the anti-fouling property after the surface was warmed to room temperature.

2. Durability tests after extremely hot environment. A 104A lubricated SLIPS surface was placed onto a hot plate $\left(200^{\circ} \mathrm{C}\right)$ for more than $1 \mathrm{~min}$, and then water and cooking oil were 
positioned onto the hot SLIPS surface. The SLIPS surface was tilted after a few seconds to test the self-cleaning properties at high temperature.

3. The relationship between heating time and liquid repellent properties. Three SLIPS glass samples (FC-70, Krytox 100B and Krytox 104A were used as lubricants respectively) were put onto a hot plate $\left(98 \pm 2{ }^{\circ} \mathrm{C}\right)$ to heat up for 5 minutes, and then these samples were cooled down to room temperature to measure the weight and CAH (of water, coffee, red wine, and corn oil) respectively; this is defined as one cycle of the hot durability tests. The samples were tested with multi cycles until the lubricants were evaporated.

4. Reversible transition between SH and SLIPS states: When a lubricant dried up in the hot durability tests, the weight, $\mathrm{CA}$ and $\mathrm{CAH}$ of water on the sample were measured respectively; then the sample was lubricated again to do the same tests of weight, $\mathrm{CA}$ and $\mathrm{CAH}$ to characterize its wettability; this is defined as one cycle of the transition tests (in this test, FC70 was used as the lubricant as an example).

\section{Mechanical robustness tests:}

In this section, all the coatings were bonded on the glass substrates using double-sided-tapes to improve the robustness.

1. Knife cut tests: FC-70, 100B and 104A lubricated samples were cut by a knife, then corn oil, red wine, coffee, and water were dropped on each sample, respectively.

2. Newton meter press: the Newton meter was used in a 'push' mode with a circle bottom area (16.2 $\mathrm{mm}$ in diameter), FC-70, 100B and 104A lubricated samples were vertically pressed by the Newton meter with forces of $2.6 \mathrm{~N}$ (the weight of the Newton meter, to give a better understanding of how big these forces are), $25 \mathrm{~N}, 50 \mathrm{~N}, 75 \mathrm{~N}, 100 \mathrm{~N}, 125 \mathrm{~N}, 150 \mathrm{~N}$, and $175 \mathrm{~N}$. For safety purposes, we used $175 \mathrm{~N}$ as the largest force in this experiment because forces larger than $175 \mathrm{~N}$ would break the glass substrates. Give the bottom area of the Newton meter $\left(2.06 \times 10^{-4} \mathrm{~m}^{2}\right)$, pressure could be calculated. Sliding angles (SA) of water and oil droplets were then measured on the surfaces before and after each press, and this is to quantify the mechanical robustness of the SLIPS surfaces.

3. Scotch tape peeling test. The Scotch tape was adhered to the FC-70, 100B and 104A lubricated samples, respectively, and then was removed. Sliding angles were measured on these samples before and after the scotch tape peeling tests. The Scotch tape was purchased from Office Depot, Inc., The Netherlands.

\section{Chemical durability tests:}

The acid $\left(\mathrm{H}_{2} \mathrm{SO}_{4}\right.$ aqueous solutions, dyed with crystal violet) and base $(\mathrm{NaOH}$ aqueous solutions, dyed with acridine orange) with different $\mathrm{pH}$ values $(\mathrm{pH}=0,2,4,6,8,10,12,14$, verified by a $\mathrm{pH}$ meter - HANNA, HI9124) were prepared for chemical durability tests. Double-sided adhesives were used to bond the glass substrates and the slippery coating throughout these tests. 
1. Acid/base droplets dropping tests: FC-70, 100B and 104A lubricated samples were initially scratched by a knife to show the mechanical robustness, and then liquids with $\mathrm{pH}$ values from 0 to 14 were dropped onto these samples that were tilted for $\sim 28^{\circ}$.

2. Acid-base neutralization on SLIPS surfaces: Acid $(\mathrm{pH}=0)$ and base $(\mathrm{pH}=14)$ droplets were positioned onto the FC-70, 100B and 104A lubricated samples, respectively. Then the droplets were guided by a knife to mix and neutralize. While acid-base neutralization, the droplet was allowed to travel around on the SLIPS surfaces and finally slid off.

3. Chemical etching test using salt solution. In this test, two plates of mild steel (that were purchased from Goodman) were used as substrates. $0.5 \mathrm{~mol} \mathrm{~L}^{-1} \mathrm{CuSO}_{4}$ aqueous solution was used as corrosive liquids. In terms of the two mild steel plates, one was untreated, the other was treated with SH paint via dip-coating, followed by lubricating with 104A. Two droplets of the corrosive liquid were positioned onto the two samples for $10 \mathrm{~min}$ to test the anticorrosion properties and chemical stability of the SLIPS coatings.

\section{Thermal, mechanical and chemical durability tests on one single sample:}

One single sample was prepared for thermal, mechanical and chemical durability tests. The sample was fabricated using double-sided-tape to bond the glass substrate and SH coating, followed by 104A lubricating.

1. Thermal test: The sample was placed onto the hot plate $\left(200^{\circ} \mathrm{C}\right)$ for more than $1 \mathrm{~min}$.

2. Mechanical test: After the thermal test, the sample was scratched by a knife followed by a Newton meter press at a force of $175 \mathrm{~N}$.

3. Chemical test: After the mechanical test, acid $(\mathrm{pH}=0)$ and base $(\mathrm{pH}=14)$ droplets were placed on the sample, and then guided to neutralize.

4. Oil repellency test: After the chemical test, a corn oil droplet was placed onto the sample surface to test its repellency to oil.

\section{References}

1 Barthlott, W. \& Neinhuis, C. Purity of the sacred lotus, or escape from contamination in biological surfaces. Planta 202, 1-8 (1997).

2 Ma, M. \& Hill, R. M. Superhydrophobic surfaces. Curr. Opin. Colloid Interface Sci. 11, 193-202 (2006).

3 Onda, T., Shibuichi, S., Satoh, N. \& Tsujii, K. Super-water-repellent fractal surfaces. Langmuir 12, 2125-2127 (1996).

$4 \quad$ Tuteja, A. et al. Designing superoleophobic surfaces. Science 318, 1618-1622 (2007).

5 Liu, T. \& Kim, C. J. Turning a surface superrepellent even to completely wetting liquids. Science 346 (2014).

$6 \quad$ Lu, Y. et al. Robust self-cleaning surfaces that function when exposed to either air or oil. Science 347, 1132-1135 (2015).

7 Wong, T.-S. et al. Bioinspired self-repairing slippery surfaces with pressure-stable omniphobicity. Nature 477, 443-447 (2011). 
Bohn, H. F. \& Federle, W. Insect aquaplaning: Nepenthes pitcher plants capture prey with the peristome, a fully wettable water-lubricated anisotropic surface. Proc. Natl. Acad. Sci. USA 101, 14138-14143 (2004).

9 Kim, P., Kreder, M. J., Alvarenga, J., Aizenberg, J. Hierarchical or not? Effect of the length scale and hierarchy of the surface roughness on omniphobicity of lubricant-infused substrates. Nano Lett. 13, 1793-1799 (2013). Hao, C. et al. Electrowetting on liquid-infused film (EWOLF): Complete reversibility and controlled droplet oscillation suppression for fast optical imaging. Sci Rep. 4, 6846 (2014).

11 Leslie, D. C. et al. A bioinspired omniphobic surface coating on medical devices prevents thrombosis and biofouling. Nature Biotechnol. 32, 1134-1140 (2014).

12 Xiao, L. et al. Slippery liquid-infused porous surfaces showing marine antibiofouling properties. ACS Appl. Mater. Interfaces 5, 10074-10080 (2013).

13 Epstein, A. K., Wong, T.-S., Belisle, R. A., Boggs, E. M. \& Aizenberg, J. Liquid-infused structured surfaces with exceptional anti-biofouling performance. Proc. Natl. Acad. Sci. USA 109, 13182-13187 (2012).

14 Liu, Q. et al. Durability of a lubricant-infused Electrospray Silicon Rubber surface as an antiicing coating. Appl. Surf. Sci. 346, 68-76 (2015).

15 Chen, J. et al. Robust prototypical anti-icing coatings with a self-lubricating liquid water layer between ice and substrate. ACS Appl. Mater. Interfaces 5, 4026-4030 (2013).

16 Wang, P., Lu, Z. \& Zhang, D. Slippery liquid-infused porous surfaces fabricated on aluminum as a barrier to corrosion induced by sulfate reducing bacteria. Corros. Sci. 93, 159-166 (2015).

17 Yang, S. et al. Slippery liquid-infused porous surface based on perfluorinated lubricant/iron tetradecanoate: Preparation and corrosion protection application. Appl. Surf. Sci. 328, 491500 (2015).

18 Nosonovsky, M. Materials science: Slippery when wetted. Nature 477, 412-413 (2011).

19 Wang, N. et al. Design and Fabrication of the Lyophobic Slippery Surface and Its Application in Anti-Icing, J. Phys. Chem. C 120, 11054-11059 (2016).

20 Manna, U. \& Lynn, D. M. Fabrication of Liquid - Infused Surfaces Using Reactive Polymer Multilayers: Principles for Manipulating the Behaviors and Mobilities of Aqueous Fluids on Slippery Liquid Interfaces. Adv. Mater. 27, 3007-3012 (2015).

21 Tesler, A. B. et al. Extremely durable biofouling-resistant metallic surfaces based on electrodeposited nanoporous tungstite films on steel. Nature Commun. 6, 8649 (2015).

22 Wang, P., Zhang, D., Lu, Z. \& Sun, S. Fabrication of slippery lubricant-infused porous surface for inhibition of microbially influenced corrosion. ACS Appl. Mater. Interfaces 8, 1120-1127 (2016).

23 Zhang, J. et al. Evaporation-induced transition from nepenthes pitcher-inspired slippery surfaces to lotus leaf-inspired superoleophobic surfaces. Langmuir 30, 14292-14299 (2014).

24 Hao, C. et al. Superhydrophobic-like tunable droplet bouncing on slippery liquid interfaces. Nature Commun. 6, 7986 (2015).

25 Miranda, D. F. et al. Physically and chemically stable ionic liquid-infused textured surfaces showing excellent dynamic omniphobicity. APL Materials 2, 056108 (2014).

26 Daniel, D., Mankin, M. N., Belisle, R. A., Wong, T.-S. \& Aizenberg, J. Lubricant-infused micro/nano-structured surfaces with tunable dynamic omniphobicity at high temperatures. Appl. Phys. Lett. 102, 231603 (2013).

27 Kim, P. et al. Liquid-infused nanostructured surfaces with extreme anti-ice and anti-frost performance. ACS nano 6, 6569-6577 (2012).

28 Hao, C. et al. Bioinspired materials: bioinspired interfacial materials with enhanced drop mobility: from fundamentals to multifunctional applications. Small 12, 1824 (2016).

29 Subramanyam, S. B., Rykaczewski, K. \& Varanasi, K. K. Ice adhesion on lubricant-impregnated textured surfaces. Langmuir 29, 13414-13418 (2013). 
30 Yu, L., Chen, G. Y., Xu, H. \& Liu, X. Substrate-Independent, Transparent Oil-Repellent Coatings with Self-Healing and Persistent Easy-Sliding Oil Repellency. ACS Nano 10, 10761085 (2016).

31 Ganesh, V. A. et al. One-step fabrication of robust and optically transparent slippery coatings. RSC Adv. 4, 55263-55270 (2014).

32 Song, T. et al. Multiple sheet-layered super slippery surfaces based on anodic aluminium oxide and its anticorrosion property. RSC Adv. 5, 70080-70085 (2015).

33 Zheng, W. T. et al. Reactive magnetron sputter deposited CNx: effects of $\mathrm{N}_{2}$ pressure and growth temperature on film composition, bonding, and microstructure. J. Vac. Sci. Technol. A 14, 2696-2701 (1996).

34 Chaussé, A. et al. The electrochemical reduction of diazonium salts on iron electrodes. The formation of covalently bonded organic layers and their effect on corrosion. Chem. Mater. 14, 392-400 (2002).

35 Ohsaka, T., Izumi, F. \& Fujiki, Y. Raman spectrum of anatase, $\mathrm{TiO}_{2}$. J. Raman Spectrosc. 7, 321-324 (1978).

$36 \mathrm{Xu}$, W., Song, J., Sun, J., Lu, Y. \& Yu, Z. Rapid fabrication of large-area, corrosion-resistant superhydrophobic Mg alloy surfaces. ACS Appl. Mater. Interfaces 3, 4404-4414 (2011).

37 Lu, Y., Sathasivam, S., Song, J., Chen, F., Xu, W., Carmalt, C. J., Parkin I. P. Creating superhydrophobic mild steel surfaces for water proofing and oil-water separation. J. Mater. Chem. A 2, 11628-11634 (2014).

38 Lu, Y., Sathasivam, S., Song, J., Xu, W., Carmalt, C. J., Parkin I. P. Water droplets bouncing on superhydrophobic soft porous materials. J. Mater. Chem. A 2, 12177-12184 (2014).

39 Krishnan, A. et al. An evaluation of methods for contact angle measurement. Colloids Surf. $B$ 43, 95-98 (2005). 
Figures

(a)

(b)

\section{Substrate}
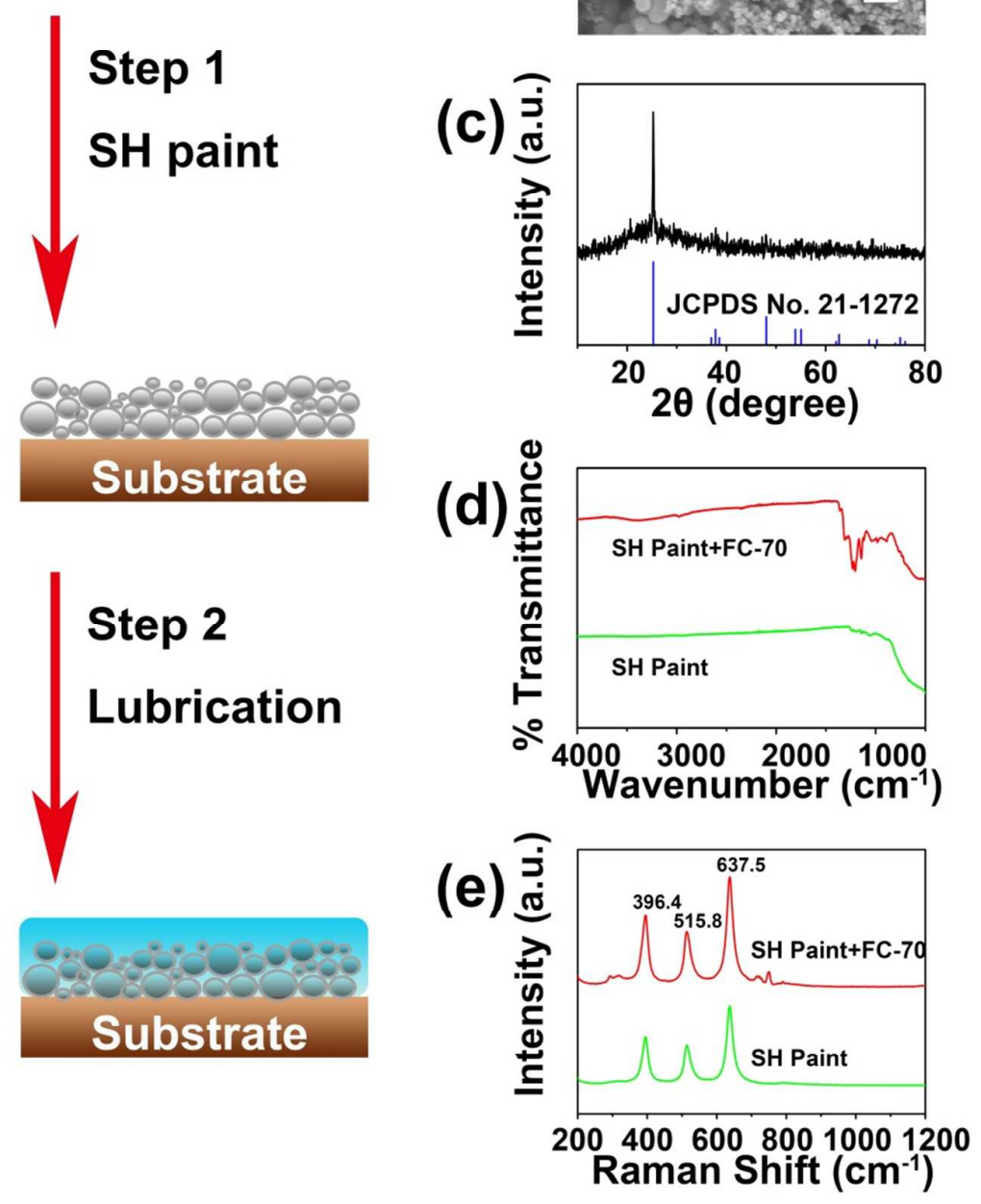

Figure 1 (a) Schematic diagram showing fabrication of the SLIPS surfaces in two steps. (b) SEM images of the SH paint treated surface. (c) XRD patterns of the SH paint. (d) FTIR spectra of the lubricated and original SH paints. (e) Raman spectra of the lubricated and original SH paints. 


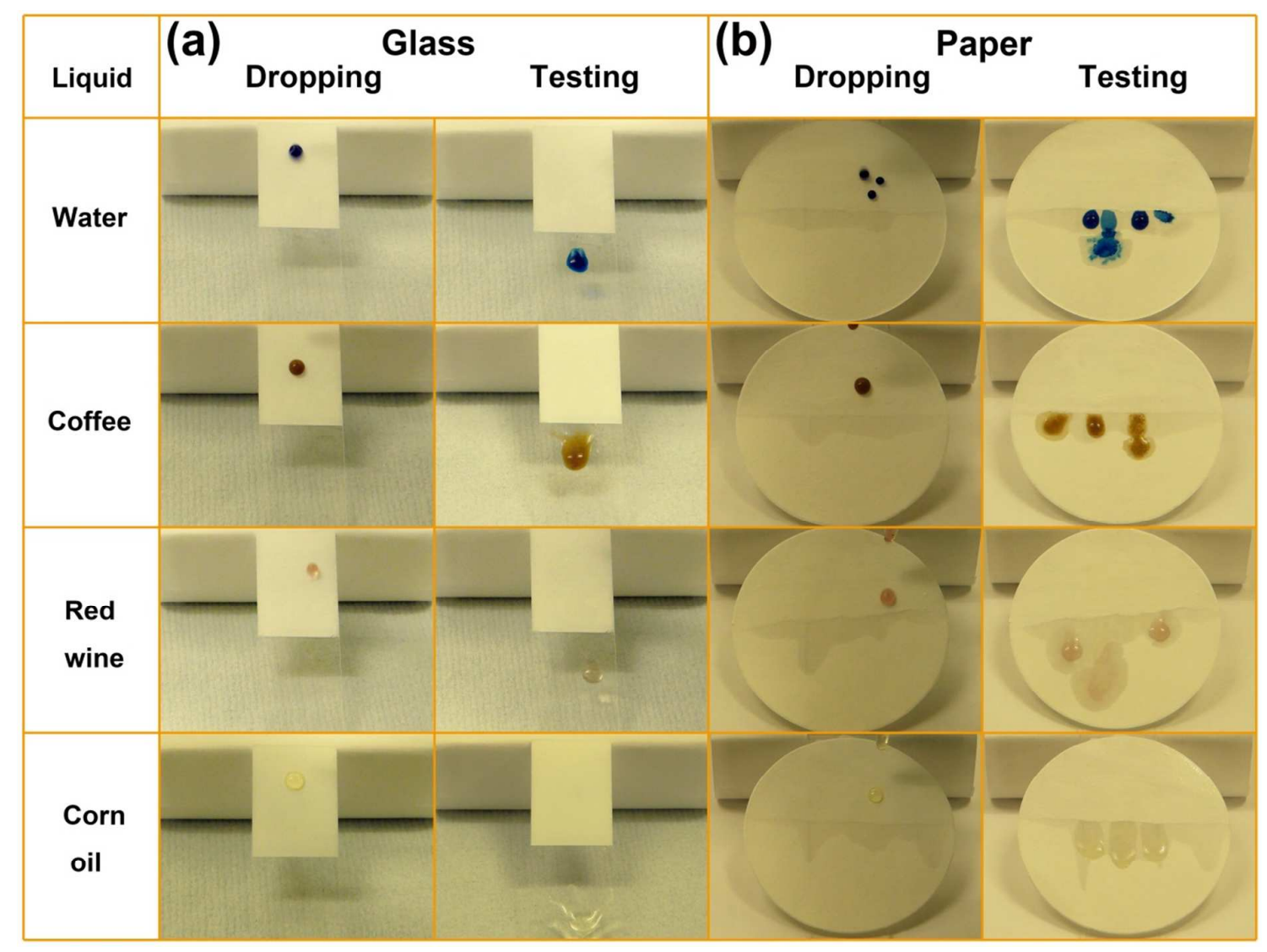

Figure 2 Liquid contaminating tests on the (a) SLIPS glass surfaces and (b) SLIPS filter paper surfaces. The upper parts were coated while the lower parts were original. 
(a) Liquid Nitrogen Test

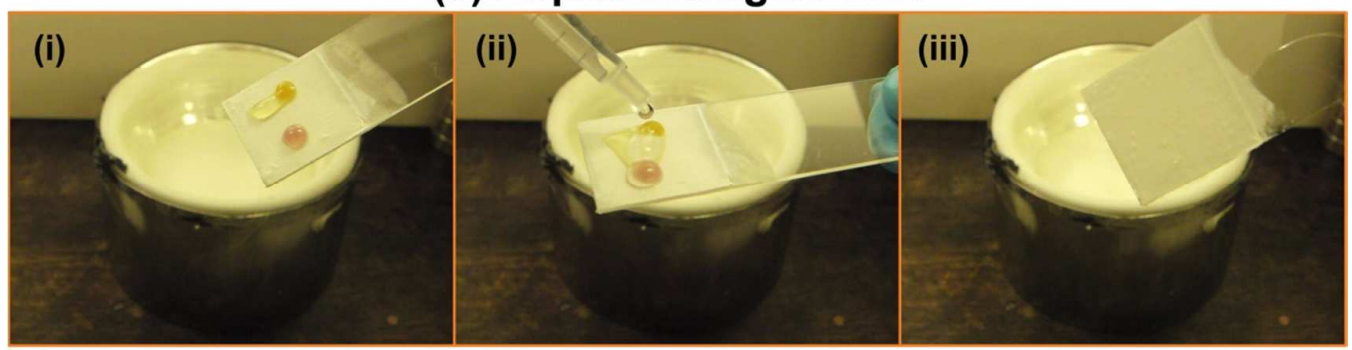

(b) Hot Plate Heating

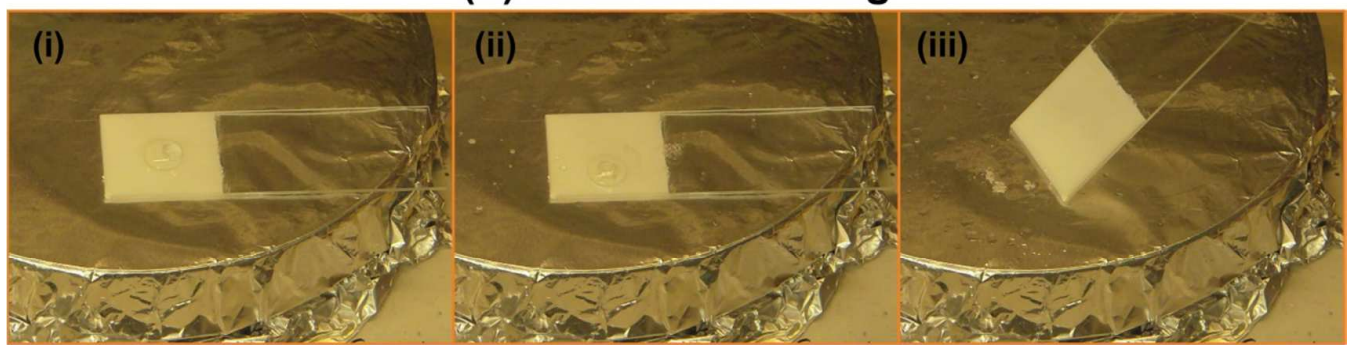

(c) Heating FC-70 and 100B samples

(d) Heating 104A

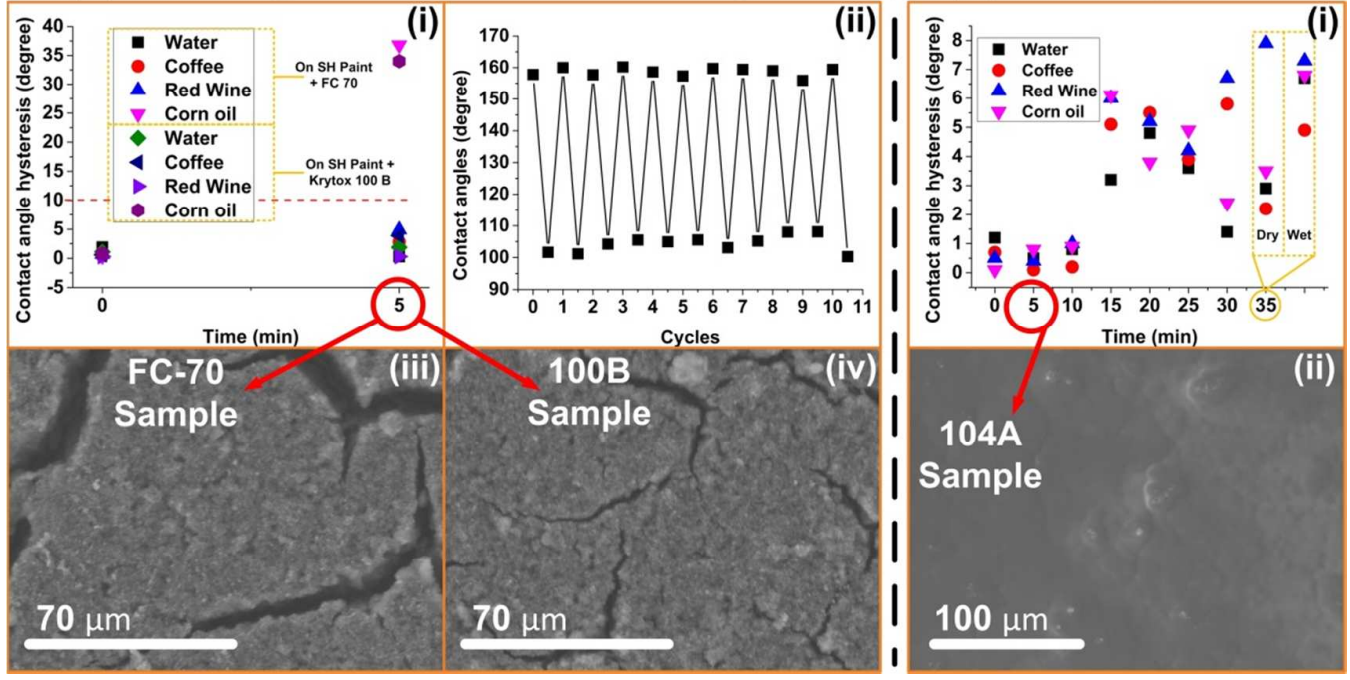

Figure 3 (a) The SLIPS glass surface (FC-70 lubricated) was inserted into liquid nitrogen to test durability. (i) Coffee (yellow), red wine (light red) and corn oil (light yellow) drops were placed on the liquid nitrogen treated SLIPS surface, and these drops firstly got frozen; (ii) water was then dropped on the surface with frozen liquids to aid their melting; (iii) The SLIPS surface melted and the liquids slid off leaving the surface clean. (b) The SLIPS glass surface (104A lubricated) was positioned on a hot plate $\left(200{ }^{\circ} \mathrm{C}\right.$ ) for $1 \mathrm{~min}$ to test its omniphobicity. (i) Water and corn oil were dropped on the hot sample (the water droplet was surrounded by oil); (ii) the water-oil mixture was "fried" to splash; (iii) the surface remained clean when tilted. (c) Heating FC-70 and 100B samples at $100^{\circ} \mathrm{C}$. (i) $\mathrm{CAH}$ of four liquids on respective FC-70 and Krytox 100B lubricated SLIPS surfaces before and after $5 \mathrm{~min}$ of heating; (ii) CA of water in the multi-cycle reversible transition between SH and SLIPS states; ESEM images of (iii) FC-70 and (iv) $100 \mathrm{~B}$ lubricated samples after heating for $5 \mathrm{~min}$ at $100{ }^{\circ} \mathrm{C}$. (d) Heating 104A sample at $100{ }^{\circ} \mathrm{C}$. (i) $\mathrm{CAH}$ of four liquids on Krytox $104 \mathrm{~A}$ lubricated SLIPS surfaces that were heated from 0 to $35 \mathrm{~min}$; (ii) ESEM images of $104 \mathrm{~A}$ lubricated samples after heating for $5 \mathrm{~min}$ at $100^{\circ} \mathrm{C}$. 


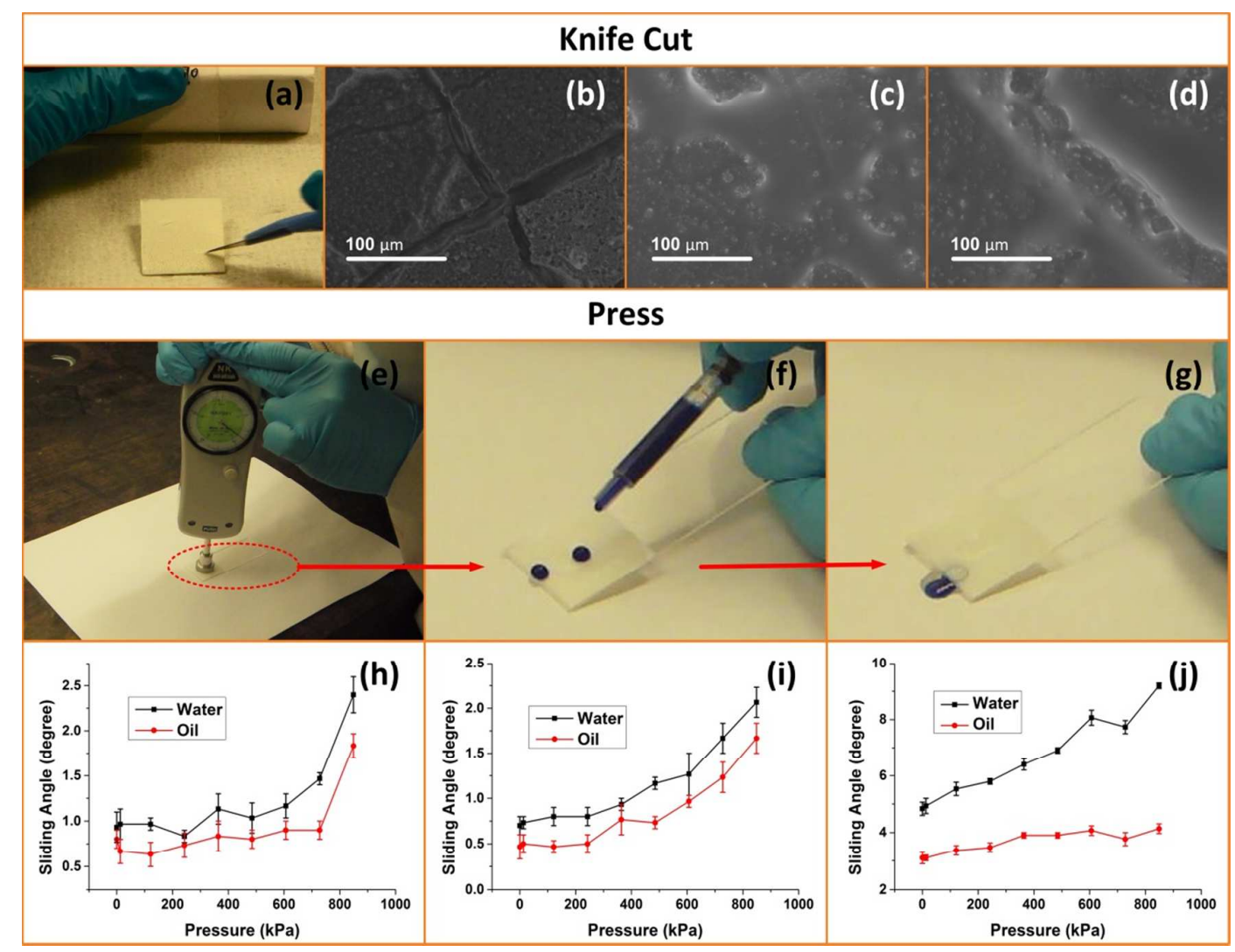

Figure 4 Mechanical robustness tests. (a) Knife cut of a FC-70 lubricated sample. (b)-(d) ESEM images of knife scratched (b) FC-70, (c) 100B and (d) 104A lubricated samples. (e) A FC-70 lubricated sample was vertically pressed by a Newton meter for $175 \mathrm{~N}(\sim 850 \mathrm{kPa})$. (f) Water and (g) oil slid off the pressed sample as shown in (e). (h)-(j) Plot of the vertical pressure and (water and oil) SAs of the pressed (h) FC-70, (i) 100B and (j) 104A lubricated samples. 


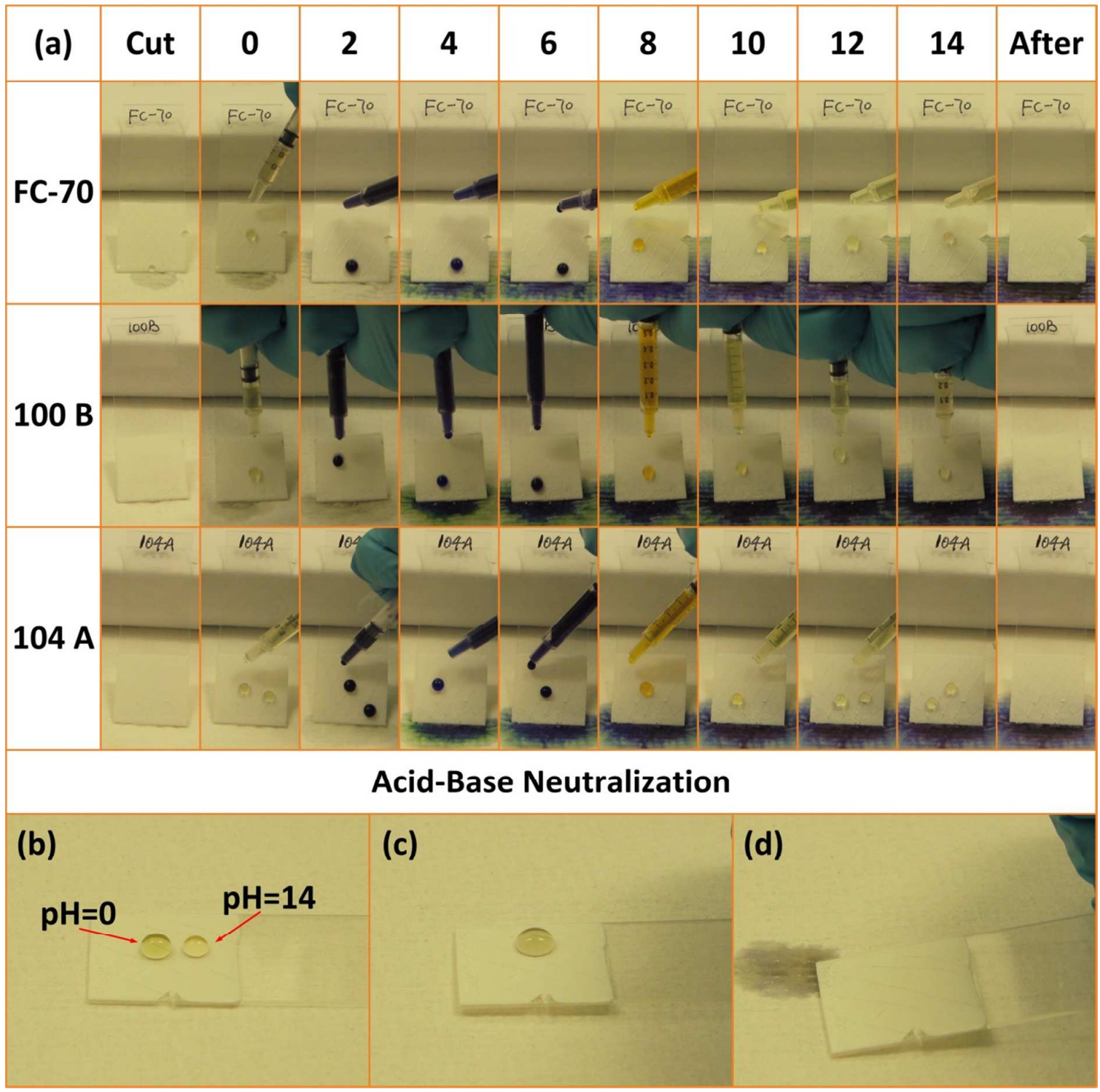

Figure 5 Chemical durability tests. (a) FC-70, 100B and 104A lubricated samples were firstly cut by a knife and then tested with water droplets with different $\mathrm{pH}$ values $(0$ 14). (b)-(d) Acid-base neutralization on a scratched FC-70 lubricated sample. (b) Acid and base droplets were placed on the surface, (c) they were guided to combine to neutralization, and then (d) the droplet freely travelled around the surface and finally slid off. 


\section{Acknowledgements}

The authors thank Paul Morris from the GBR Technology Limited for the donation of the lubricants of Krytox 100B and 104A. Thanks to Dr. Tom Gregory for ESEM training.

\section{Author Contributions Statement}

Y.L. developed the initial ideas, designed the experiments and organised the work; Y.L. and G.H. performed the experiments and characterizations; I.P.P. and C.J.C supervised the work; Y.L. and I.P.P. wrote the paper and all authors provided comments.

\section{Additional Information}

\section{Supplementary information}

Supporting documents and movies regarding the characterization and functional tests are available here.

Competing financial interests: The authors have filed a patent on this work. 
Supporting information for

\section{Synthesis and characterization of omniphobic surfaces with thermal, mechanical and chemical stability}

Yao Lu, Guanjie He*, Claire J. Carmalt, Ivan P. Parkin*

Department of Chemistry, University College London, 20 Gordon Street, London, WC1H 0AJ, UK

I.P.P.: i.p.parkin@ucl.ac.uk; G. H.: guanjie.he.14@ucl.ac.uk

\section{Supporting Tables and Figures}

Supplementary Table S1. Viscosities (cST) of lubricants at different temperatures and boiling points $(1 \mathrm{~atm})$ and estimated useful range (UR, based on pour point and where evaporation is approximately $10 \%$ ) are according to the respective product information[1,2]. The densities of lubricants at room temperature $\left(\sim 20{ }^{\circ} \mathrm{C}\right)$ were calculated by weighing the mass of a $1 \mathrm{ml}$ volume of the lubricants using an analytical balance Ohaus; $\delta=0.01 \mathrm{mg}$.

\begin{tabular}{c|c|c|c}
\hline Temperature $\left({ }^{\circ} \mathrm{C}\right)$ & Fluorinert FC-70 & Krytox 100B & Krytox 104A \\
\hline 20 & $\sim 18$ & 12.4 & 177 \\
\hline 40 & $\sim 5.6$ & 5.5 & 60 \\
\hline 100 & $\sim 1.03$ & - & 8.4 \\
\hline $\begin{array}{c}\text { Boiling points/UR } \\
\left({ }^{\circ} \mathrm{C}\right)\end{array}$ & 215 & $-70-66$ & $-51-179$ \\
\hline $\begin{array}{c}\text { Density at room } \\
\text { temperature }\left(\mathrm{g} / \mathrm{cm}^{3}\right)\end{array}$ & 2.01042 & 1.84292 & 1.85270 \\
\hline
\end{tabular}




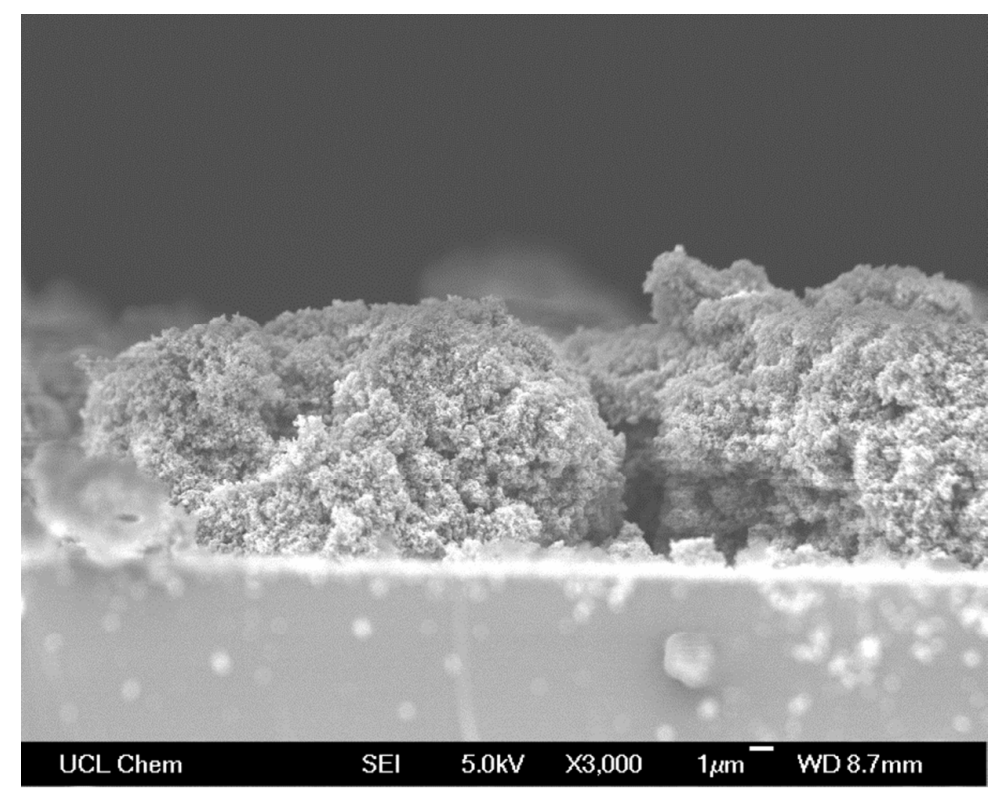

Supplementary Fig. S1. Cross-sectional SEM image of the SH paint treated glass substrate. The thickness of the SH coating was $\sim 14 \pm 1 \mu \mathrm{m}$.

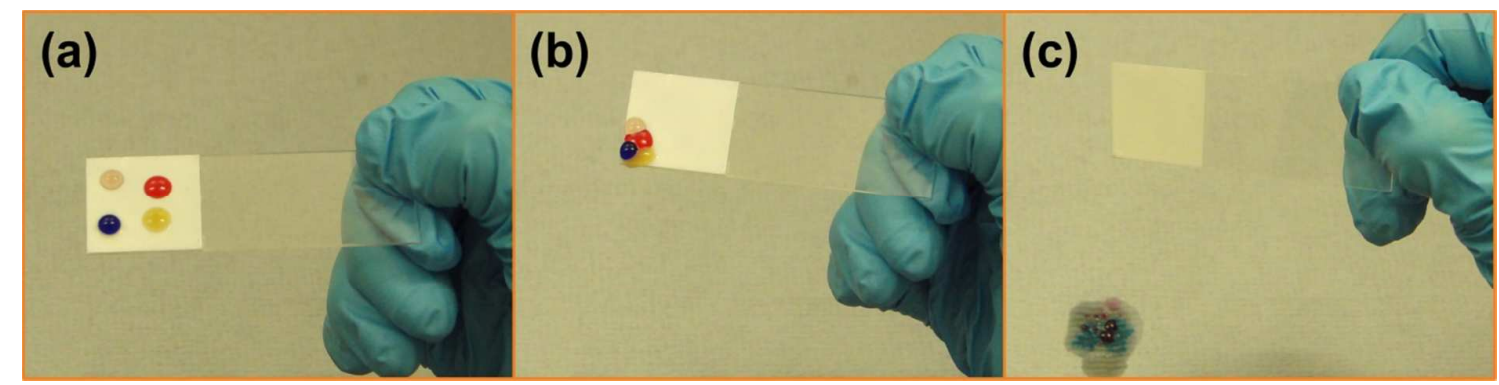

Supplementary Fig. S2. Four liquids contaminating tests on a SLIPS glass surface, the liquids are hexadecane (red), red wine (light red), coffee (yellow) and water (blue), respectively. (a) Liquids were staying on the surface and moving slowly. (b) Liquids got together and tended to slide off. (c) Liquids slid off leaving the surface clean. 


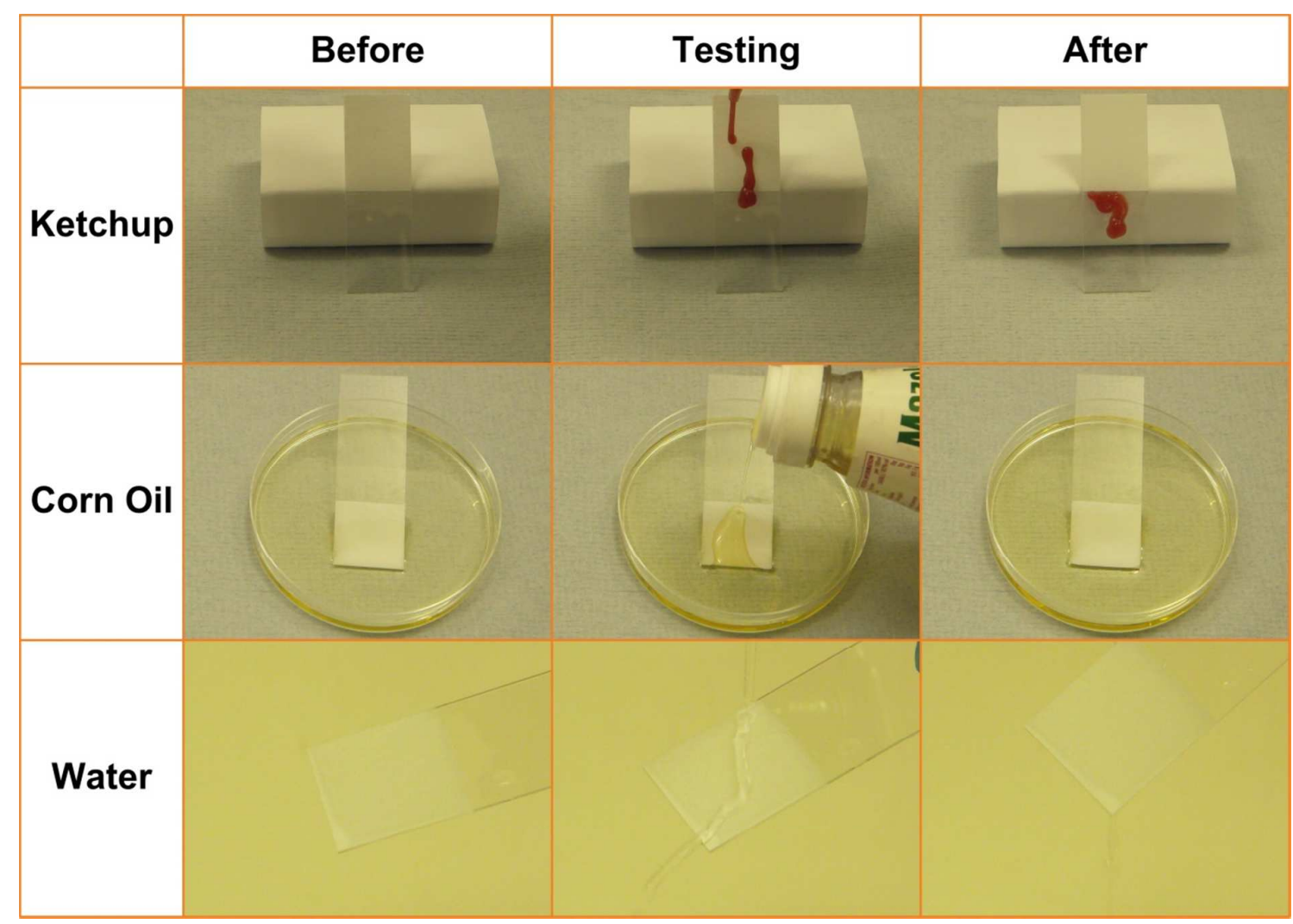

Supplementary Fig. S3. Contamination-repellent tests on the SLIPS surfaces using ketchup, running corn oil and tapped water. 
Supplementary Table S2. CA of water, weight of samples and the thickness of the FC-70 lubricating layer in the multi-cycle reversible transition tests (the treated surface area is 25 $\mathrm{mm} \times 28 \mathrm{~mm})$.

\begin{tabular}{|c|c|c|c|}
\hline Data & CA & Weight & Thickness \\
$(\mathrm{g})$ & $(\mu \mathrm{m})$
\end{tabular}


Supplementary Table S3. Mass (g) of the Fluorinert FC-70, Krytox 100B and Krytox 104A lubricated samples and the corresponding average thickness $(\mu \mathrm{m})$ of the lubricating layers as the increase of the heating time (the treated surface area is $25 \mathrm{~mm} \times 25 \mathrm{~mm}$ ).

\begin{tabular}{|l|c|c|c|}
\hline \multicolumn{1}{|c|}{ Sample } & $\begin{array}{c}\text { Fluorinert FC-70 } \\
\text { Weight/Thickness }\end{array}$ & $\begin{array}{c}\text { Krytox 100B } \\
\text { Weight/Thickness }\end{array}$ & $\begin{array}{c}\text { Krytox 104A } \\
\text { Weight/Thickness }\end{array}$ \\
\hline Before lubricants & $4.5423 / 0$ & $4.5575 / 0$ & $4.5239 / 0$ \\
\hline After lubricants & $4.6522 / 87.464$ & $4.6619 / 90.639$ & $4.7543 / 198.974$ \\
\hline After 5 min & $4.5421 / 0$ & $4.5571 / 0$ & $4.6659 / 122.632$ \\
\hline After 10 min & - & - & $4.6314 / 92.837$ \\
\hline After 15 min & - & - & $4.5908 / 57.775$ \\
\hline After 20 min & - & - & $4.5862 / 53.803$ \\
\hline After 25 min & - & - & $4.5659 / 36.271$ \\
\hline After 30 min & - & - & $4.5532 / 25.304$ \\
\hline After 35 min & - & - & \\
\hline
\end{tabular}

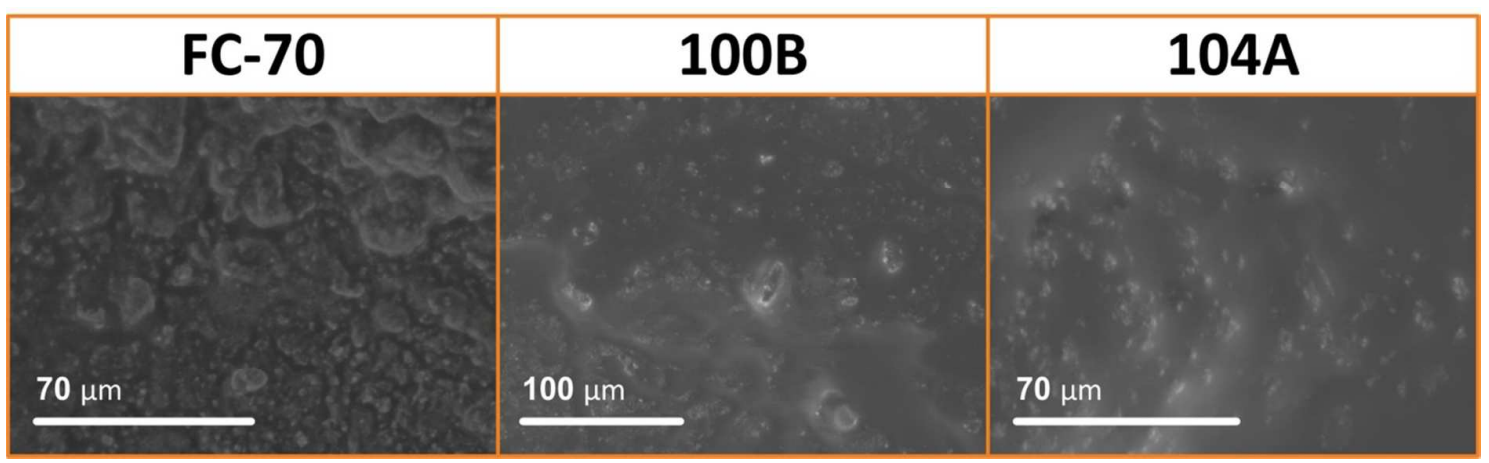

Supplementary Fig. S4. ESEM images of FC-70, 100B and 104A lubricated SLIPS surfaces. It is shown that lubricants were embedded in the SH surface structures. 


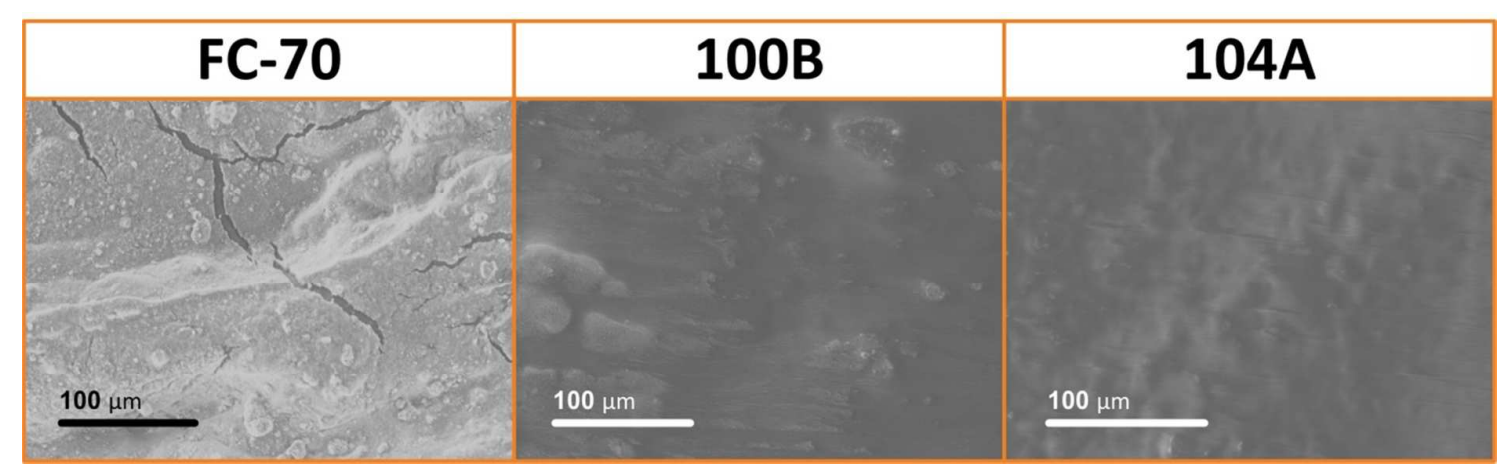

Supplementary Fig. S5. ESEM images of FC-70, 100B and 104A lubricated SLIPS surfaces after being pressed by a force of $175 \mathrm{~N}(\sim 849.02 \mathrm{kPa})$.

Supplementary Table S4. Scotch tape peeling was used to test the surface robustness. Both water and corn oil sliding angles of FC-70, 100B and 104A lubricated SLIPS surfaces were measured before and after the scotch peeling tests. The data shown in this table is in degrees $\left({ }^{\circ}\right)$.

\begin{tabular}{|c|c|c|c|}
\hline Before/After Peeling & FC 70 & $100 \mathrm{~B}$ & $104 \mathrm{~A}$ \\
\hline Water & $1.1 \pm 0.1 / 2.0 \pm 0.1$ & $0.8 \pm 0.1 / 1.5 \pm 0.1$ & $4.9 \pm 0.2 / 5.1 \pm 0.2$ \\
\hline Corn Oil & $1.0 \pm 0.2 / 1.3 \pm 0.1$ & $0.6 \pm 0.1 / 0.7 \pm 0.1$ & $2.9 \pm 0.2 / 3.5 \pm 0.2$ \\
\hline
\end{tabular}

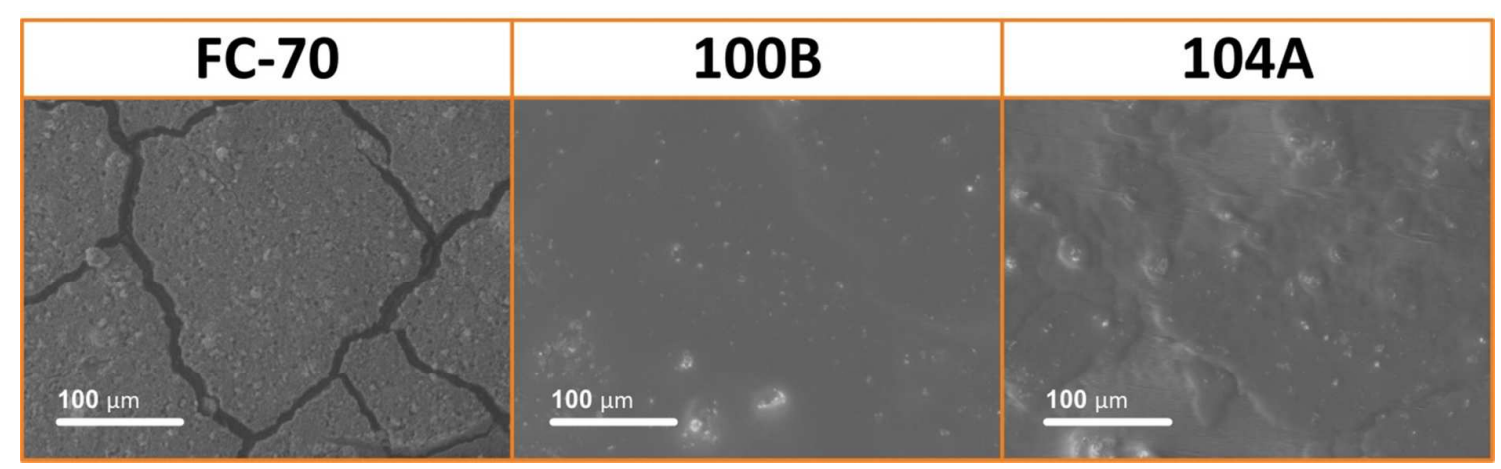

Supplementary Fig. S6. ESEM images of FC-70, 100B and 104A lubricated SLIPS surfaces after the acid-base neutralization. 

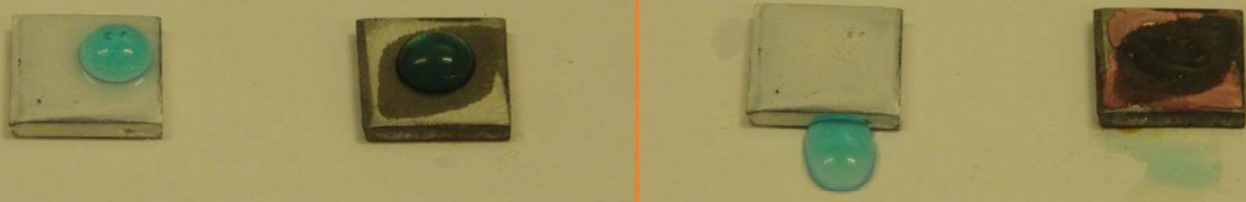

$0 \mathrm{~min}$

\section{$10 \mathrm{~min}$ later}

Supplementary Fig. S7. Droplets of $0.5 \mathrm{~mol} \mathrm{~L}^{-1} \mathrm{CuSO}_{4}$ aqueous solution were positioned onto untreated mild steel and 104A lubricated SLIPS mild steel, respectively. After 10 minutes, the untreated sample was chemically etched, while the SLIPS coated mild steel did not show any traces of corrosions.

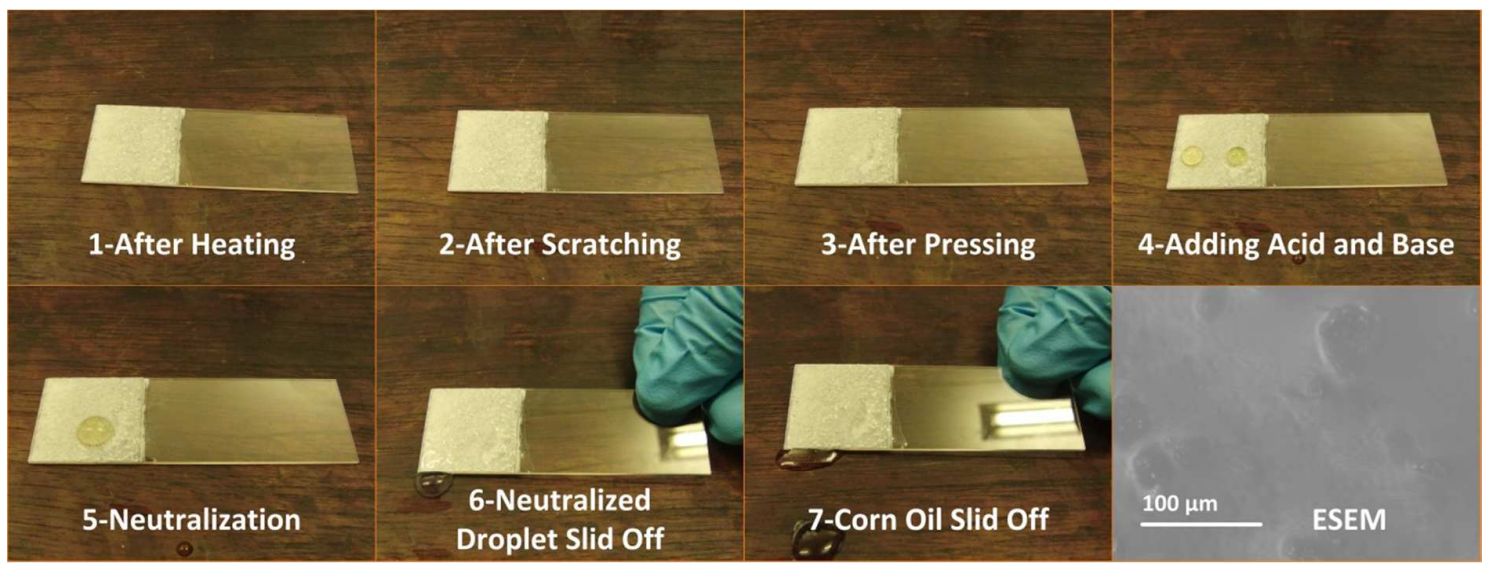

Supplementary Fig. S8. Thermal, mechanical and chemical durability tests on one single sample. The sample was heated at $200{ }^{\circ} \mathrm{C}$, scratched, pressed at $175 \mathrm{~N}$, and then acid (the right droplet, $\mathrm{pH}=0$ ) and base (the left droplet, $\mathrm{pH}=14$ ) were positioned on the sample to neutralize. After these tests, a corn oil droplet was still able to slide off, indicating the surface retained slippery and omniphobic. ESEM image shows that the surface did not significantly change after these tests, indicating that the SLIPS surface is thermally, mechanically and chemically durable. 


\section{Movie captions}

\section{Supplementary Movie S1}

Water (dyed blue), coffee, red wine and corn oil were dropped onto the treated SLIPS glass surfaces to compare with the untreated parts and to test the self-cleaning ability.

\section{Supplementary Movie S2}

Water (dyed blue), coffee, red wine and corn oil were dropped onto the treated SLIPS filter paper surfaces to compare with the untreated parts and to test the self-cleaning ability. This is to show that the method of fabricating SLIPS surfaces can be applied to different substrates.

\section{Supplementary Movie S3}

Hexadecane, red wine, coffee and water droplets slid around and finally fell off the SLIPS surface. This is to show that the surface was still slippery and omniphobic when impacting different liquids together for some time.

\section{Supplementary Movie S4}

Tomato ketchup was dropped onto the treated SLIPS glass surfaces to compare with the untreated parts and to test the self-cleaning ability.

\section{Supplementary Movie S5}

Running and continuous liquid (corn oil and water) contaminations were applied to test the self-cleaning properties of the SLIPS surfaces.

\section{Supplementary Movie S6}

Recovery tests of the SLIPS surface after being inserted into liquid nitrogen. Liquids were then dropped onto the surface to test the recovery of the omniphobic property.

\section{Supplementary Movie S7}

Water and oil droplets were positioned on a (104A lubricated) SLIPS surface which had been heating for $1 \mathrm{~min}$ at $200{ }^{\circ} \mathrm{C}$. The water-oil mixture was then fried to splash, and finally slid off and the SLIPS surface retained clean.

\section{Supplementary Movie S8}

Water, red wine, coffee, corn oil and hexadecane were dropped on the surface that was lubricated - heated to dry - lubricated for 10 cycles. This is to show that the surface retained self-cleaning properties after multi-cycles of the hot recovery tests.

\section{Supplementary Movie S9}


Knife cut tests: FC-70, 100B and 104A lubricated samples were cut by a knife followed by liquid dropping tests. The liquids were corn oil, red wine, coffee, and water, respectively, on each sample.

\section{Supplementary Movie S10}

Press tests: FC-70 lubricated sample was pressed by a Newton meter with a force of $175 \mathrm{~N}$ $(\sim 849 \mathrm{kPa})$. Then the pressed surface was tested by dropping water and oil.

\section{Supplementary Movie S11}

Acid $(\mathrm{pH}=0$, the left droplet) and base $(\mathrm{pH}=14$, the right droplet) neutralization on the FC70, 100B and 104A lubricated samples, respectively. Acid and base droplets were guided by a knife to combine, neutralize and finally slid off.

\section{References}

[1] http://multimedia.3m.com/mws/media/648910/fluorinert-electronic-liquid-fc-70.pdf.

[2]

http://www2.dupont.com/Lubricants/en US/assets/downloads/Typical Properties of Krytox GPL H -58510-3.pdf. 